\title{
Genome-wide transcriptomics of aging in the rotifer Brachionus manjavacas, an emerging model system
}

Kristin E. Gribble and David B. Mark Welch*

\begin{abstract}
Background: Understanding gene expression changes over lifespan in diverse animal species will lead to insights to conserved processes in the biology of aging and allow development of interventions to improve health. Rotifers are small aquatic invertebrates that have been used in aging studies for nearly 100 years and are now re-emerging as a modern model system. To provide a baseline to evaluate genetic responses to interventions that change health throughout lifespan and a framework for new hypotheses about the molecular genetic mechanisms of aging, we examined the transcriptome of an asexual female lineage of the rotifer Brachionus manjavacas at five life stages: eggs, neonates, and early-, late-, and post-reproductive adults.

Results: There are widespread shifts in gene expression over the lifespan of $B$. manjavacas; the largest change occurs between neonates and early reproductive adults and is characterized by down-regulation of developmental genes and up-regulation of genes involved in reproduction. The expression profile of post-reproductive adults was distinct from that of other life stages. While few genes were significantly differentially expressed in the late- to postreproductive transition, gene set enrichment analysis revealed multiple down-regulated pathways in metabolism, maintenance and repair, and proteostasis, united by genes involved in mitochondrial function and oxidative phosphorylation.

Conclusions: This study provides the first examination of changes in gene expression over lifespan in rotifers. We detected differential expression of many genes with human orthologs that are absent in Drosophila and C. elegans, highlighting the potential of the rotifer model in aging studies. Our findings suggest that small but coordinated changes in expression of many genes in pathways that integrate diverse functions drive the aging process. The observation of simultaneous declines in expression of genes in multiple pathways may have consequences for health and longevity not detected by single- or multi-gene knockdown in otherwise healthy animals. Investigation of subtle but genome-wide change in these pathways during aging is an important area for future study.
\end{abstract}

Keywords: Aging, Rotifer, Monogonont, RNA-Seq, Transcriptome

\section{Background}

Aging is a complex process characterized by the progressive degeneration of a healthy phenotype and correlated with a decline in the ability to withstand cellular stress and damage. The subject of investigation for decades, the underlying molecular genetic causes of and responses to aging remain an area of active study. Research from model systems has

\footnotetext{
* Correspondence: dmarkwelch@mbl.edu

Josephine Bay Paul Center for Comparative Molecular Biology and Evolution, Marine Biological Laboratory, Woods Hole, MA 02543, USA

characterized a range of physiological and molecular phenotypes associated with aging. These include genomic instability caused by accumulation of DNA damage, dysregulation of repair mechanisms, and telomere attrition; epigenetic alterations; dysregulation of transcription; loss of proteostasis; cellular senescence; and deregulated nutrient sensing, metabolic pathways, and energy use (reviewed in [1]). Separating causation from correlation between these phenotypes and aging remains a challenge, however.

Many of the genes and gene networks that modulate aging are conserved across animal phyla. For this reason, 
the highly tractable model systems Drosophila and Caenorhabditis have provided fundamental advances in our understanding of the genetic control of cellular processes that affect aging. There is a growing realization that increasing the evolutionary breadth in animal systems used in aging studies will lead to discovery of effects and mechanisms that are more likely to be robust and reveal fundamental principles of aging. The use of diverse models may also reveal previously unknown genetic factors involved in healthy aging in humans. The lineages leading to Drosophila melanogaster and Caenorhabditis elegans have each undergone significant genome reduction, and these standard model systems lack many vertebrate gene homologs that are present in other invertebrates [2-9]. In addition, arthropods and nematodes are more closely related to each other than originally thought $[10,11]$, limiting the evolutionary range in comparative studies of aging [12] and thus the degree to which conclusions can be reliably generalized from these models to humans.

Rotifers are small aquatic invertebrates that have been used in aging studies for nearly 100 years but have only recently been developed as a modern model system for the study of aging $[13,14]$. They are early-branching triploblast animals that have not undergone extensive genome reduction, but instead share many genes with vertebrates that are missing in flies and worms $[15,16]$. This suggests that the rotifer genome may contain genes and pathways that modulate aging in vertebrates but are not present in other invertebrate model systems.

Rotifers develop directly without a larval stage and are eutelic, without cell division after hatching except in the germline [17]. Many species can be easily cultured in water on a simple diet of bacteria or single-celled algae, and even a large experiment can be conducted in a small number of tissue culture dishes. Most have an asexual stage to their life cycle, and can be cultured asexually indefinitely, maintaining genetic identity across generations and between test conditions.

The rotifer Brachionus manjavacas has a wellcharacterized life cycle and aging phenotype. Under standard laboratory conditions, asexual B. manjavacas neonates increase in size quickly after hatching and produce their first offspring within $48 \mathrm{~h}$. Reproduction increases to a maximum of 6 offspring/day around day 5 , then declines to the end of the reproductive period on approximately day 8 ; the post-reproductive (senescent) period last for another 2-4 days. As a B. manjavacas female ages, her color changes from translucent to opaque, her foot begins to drag instead of being tucked up against the body, she cannot attach as firmly to a substrate, and swimming speed declines dramatically $[14,18]$. Median lifespan is approximately 10 days; death is characterized by a lack of motion of cilia, appendages, or internal organs and is frequently accompanied by a loss of membrane integrity.

Brachionus rotifers have recently been used to study the effects on healthspan and aging of stress [18-21]; temperature [22]; and dietary restriction and metabolism, including maternal effects [23-28]. To define the transcriptional changes associated with normal aging in this emerging model system, we conducted RNA-Seq at five timepoints over the lifespan of $B$. manjavacas, from eggs to senescence (Fig. 1). This study provides the first insights into genome-wide changes in gene expression over the lifespan of a rotifer, and continues development of this model system to study the biology of aging.

\section{Methods}

\section{Culture}

We maintained the monogonont rotifer Brachionus manjavacas in batch culture in 15 ppt Instant Ocean on a diet of the chlorophyte alga Tetraselmis sueccica. We grew $T$. sueccica in bubbled $\mathrm{f} / 2$ medium [29] prepared in 15 ppt Instant Ocean. We maintained both $B$. manjavacas and $T$. suecica at $21{ }^{\circ} \mathrm{C}$ on a light:dark cycle of $12 \mathrm{~h}: 12 \mathrm{~h}$.

\section{Sample preparation and sequencing}

One week prior to beginning the experiments, we inoculated a new batch culture of $B$. manjavacas at approximately 1 rotifer $/ 25 \mathrm{~mL}$. To prevent induction of sexual

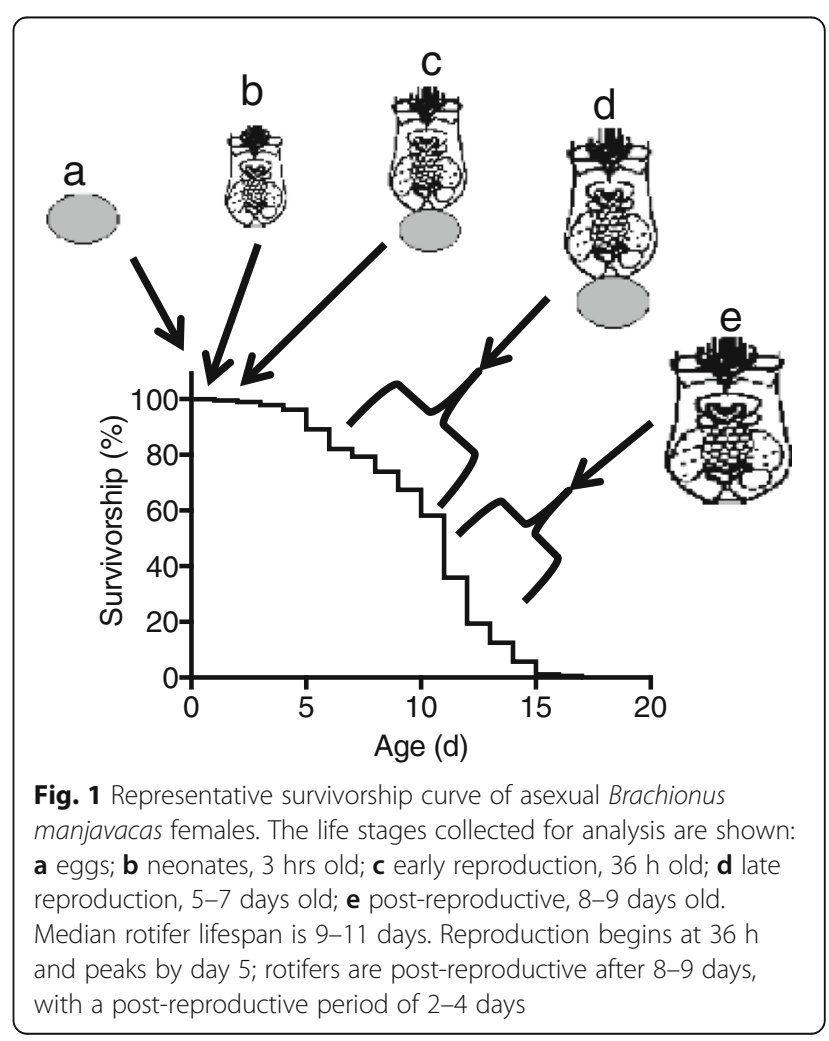


reproduction due to crowding, we maintained the culture at a density below 1 rotifer $/ 2 \mathrm{ml}$ by daily sieving and transfer into new T. suecica and medium. Concentrations of T. suecica were always above $6 \times 10^{5}$ cells $/ \mathrm{ml}$, which we previously demonstrated allows feeding ad libitum [28].

We collected samples at five time points over the lifespan of amictic B. manjavacas females (Fig. 1): eggs, neonates $1-3 \mathrm{~h}$ old, $36 \mathrm{~h}$ old early reproductive females, reproductive females of mixed ages from 3 to $6 \mathrm{~d}$ old, and post-reproductive females between 6 and 9 days old. Reproductive females carry one or more eggs, which we removed prior to harvesting by vortexing. For each time point, we harvested $100-200$ rotifers into a $1.5 \mathrm{ml}$ centrifuge tube, immobilized them using distilled water and centrifuged to a pellet. We resuspended the pellet in $150 \mu \mathrm{l}$ TriZOL reagent (Invitrogen, Waltham MA), mashed it with a pestle, brought the sample up to $1 \mathrm{~mL}$ with TriZOL reagent, and froze at $-80{ }^{\circ} \mathrm{C}$ until RNA extraction.

We constructed RNA-Seq libraries starting with total RNA extracted using the TriZOL reagent protocol, followed by removal of residual DNA using Turbo DNA-free (Ambion, Waltham, MA). We used the Ovation 3'-DGE kit (NuGEN, San Carlos, CA) to create cDNA from mRNA using linear amplification to prevent PCR bias, sheared to $400-500$ bp with Covaris, endrepaired fragments (S1 Nuclease, Promega, Madison, WI and Quick Blunting Kit, New England Biolabs, Ipswich, MA), and adenylated 5' ends using Taq (New England Biolabs). We ligated unique TruSeq compatible indexed adapters to each library and size selected to 400-600 bp using Pippin Prep (Sage Science, Beverly, MA). Illumina HiSeq sequencing generated an average of 40 million 2x100base read pairs.

We repeated the entire procedure from inoculation through sequencing for a biological replicate set of libraries, which generated an average of 34 million pairs of 100 base reads.

\section{Transcriptome assembly and annotation}

We filtered reads according to Minoche [30] and mapped high quality read pairs to our published B. manjavacas transcriptome assembled from 454 data (SRR801079; [31]) using CLC Genomics Workbench 7 (https://www.qiagenbioinformatics.com/). We then assembled the unmapped reads and unassembled 454 reads and added these contigs to the assembly. To remove transcripts from media contaminants and T. suecica, we eliminated contigs that had a top BLAST hit in the NCBI refseq protein database to a non-Metazoan with an $E$ value less than $10^{-6}$, or that had no hit with an $E$ value less than $10^{1} ; 43,149$ contigs remained. We annotated these contigs by BLAST to the KEGG,
Swissprot, and Human Protein Reference databases at an $E$ value cutoff of $10^{-10}$; we used the top hits to HPRDv9 to assign HGNC gene symbols for gene set enrichment analysis, described below. We refer to genes by HGNC gene symbol whenever possible. We used reciprocal best blastx scores to assign orthology between each transcript and all peptides from Homo sapiens (assembly GRCh38.p7), Drosophila melanogaster (assembly BDGP5), and Caenorhabditis elegans (assembly WBcel235) downloaded from www.ensembl.org. We considered a gene to be present in humans and B. manjavacas and absent in flies and worms if the $E$ value between human and rotifer was less than $10^{-20}$ and greater than $10^{-10}$ to flies or worms.

\section{Differential gene expression and pathway analysis}

We mapped quality filtered reads separately from each library to the assembly using CLC. To prevent bias toward transcripts with very low levels of expression, we confined our differential expression analysis to the 22,064 contigs with an average FPKM $\geq 1$ across all five ages and two replicates. The mean length of these contigs was $906 \mathrm{bp}$, with an N50 of $1231 \mathrm{bp}$. We determined significantly differently expressed transcripts between each life stage using baySeq [32], with an FDR cutoff of 0.05 (see Additional file 1 for baySeq R scripts). These composed the test set to find enriched Gene Ontology (GO) terms using Blast2GO. Refseq annotation was imported for the entire transcriptome of 22,064 contigs, and additional annotation with GO IDs and InterPro identifiers was conducted in Blast2GO with a permissive evalue cutoff of $10^{-3}$. Up- and down-regulated transcripts for each life stage transition were analyzed separately with all 22,064 contigs as the reference set for the Fisher's exact test (FDR $\leq 0.05$, two-tailed analysis). Significantly differently expressed GO terms were reduced to specific terms for analysis.

We conducted gene set enrichment analysis (GSEA; $[33,34])$ on pairwise consecutive time points. For input to GSEA, we annotated the B. manjavacas transcriptome with HGNC gene names as described above [35]. Transcripts with the same HUGO annotation were summed for analysis, and a constant of ten was added to all FPKM values to avoid bias toward genes with low expression when converting to $\log 2$. We searched for enrichment of KEGG pathways gene sets using our entire expression dataset, with 1000 permutations by gene set. The metric chosen for ranking genes was the $\log 2$ ratio of classes; significant enrichment, either up or down, was designated as FDR $\leq 0.25$. The normalized enrichment statistic was used to create a heat map of KEGG pathways. Leading edge analysis in GSEA was used to identify genes shared among more than one enriched gene set; genes that are differentially expressed at a given 
age and are found in several pathways are more likely to have an impact on multiple functions than are those found in only a single pathway.

For a detailed look at gene expression in pathways of interest, KEGG pathway annotation was used to select relevant transcripts, and these were clustered according to expression level with the hierarchical cluster module of GenePattern [36] using Pearson correlation of $\log 2$ transformed FPKM values.

\section{Results and discussion}

Aging in B. manjavacas is characterized by large shifts in transcript abundance between major life stages (Table 1, Figs 2, 3 and 4), which we take as a first-order proxy for gene expression. Among the 22,064 contigs representing genes with an average FPKM $>1,15,826$ genes had significant differential expression in one or more pairwise comparisons of life stages $(\mathrm{FDR}<0.05)$. Expression profiles were similar between eggs and neonates, and between early and late reproductive stages; these stages broadly represent developmental and reproductive periods, respectively. The expression profile of the post-reproductive or senescent stage was distinct from the four other life stages.

Analysis of these genes in Blast2GO revealed numerous gene ontology terms that were enriched in each life stage transition (Additional file 2). Using GSEA on all genes, we found 86 KEGG pathways enriched in at least one transition (Fig. 5) and 53 pathways enriched in two or more transitions. Results largely agreed with those found in Blast2GO, even though these methods rely on different annotation, analysis, and tests for significance. Because GSEA uses only those transcripts that can be annotated to HGNC and combines expression of transcript variants to show gene-level transcription, we also examined aging-related KEGG-defined pathways using annotation defined by refseq and swissprot.

There were 6251 genes with significant differential expression in one or more sequential life stages. Here we summarize our analyses of each life stage transition, with emphasis on gene expression changes involved in specific processes related to aging, in particular the shift from young, reproductive females to aged, senescent, post-reproductive females.

Table 1 The number of genes significantly differentially expressed between each life stage (baySeq, FDR < 0.05)

\begin{tabular}{|c|c|c|c|c|c|c|c|c|}
\hline & \multicolumn{2}{|r|}{ Eggs } & \multicolumn{2}{|c|}{ Neonates } & \multicolumn{2}{|r|}{ Early } & \multicolumn{2}{|r|}{ Late } \\
\hline & Up & Down & Up & Down & Up & Down & $\overline{U p}$ & Down \\
\hline Neonates & 1152 & 876 & - & - & - & - & - & \\
\hline Early & 3505 & 3469 & 2786 & 2718 & - & - & - & \\
\hline Late & 6945 & 5814 & 4895 & 3740 & 1140 & 236 & - & \\
\hline Post & 5266 & 4557 & 657 & 844 & 550 & 350 & 25 & 53 \\
\hline
\end{tabular}

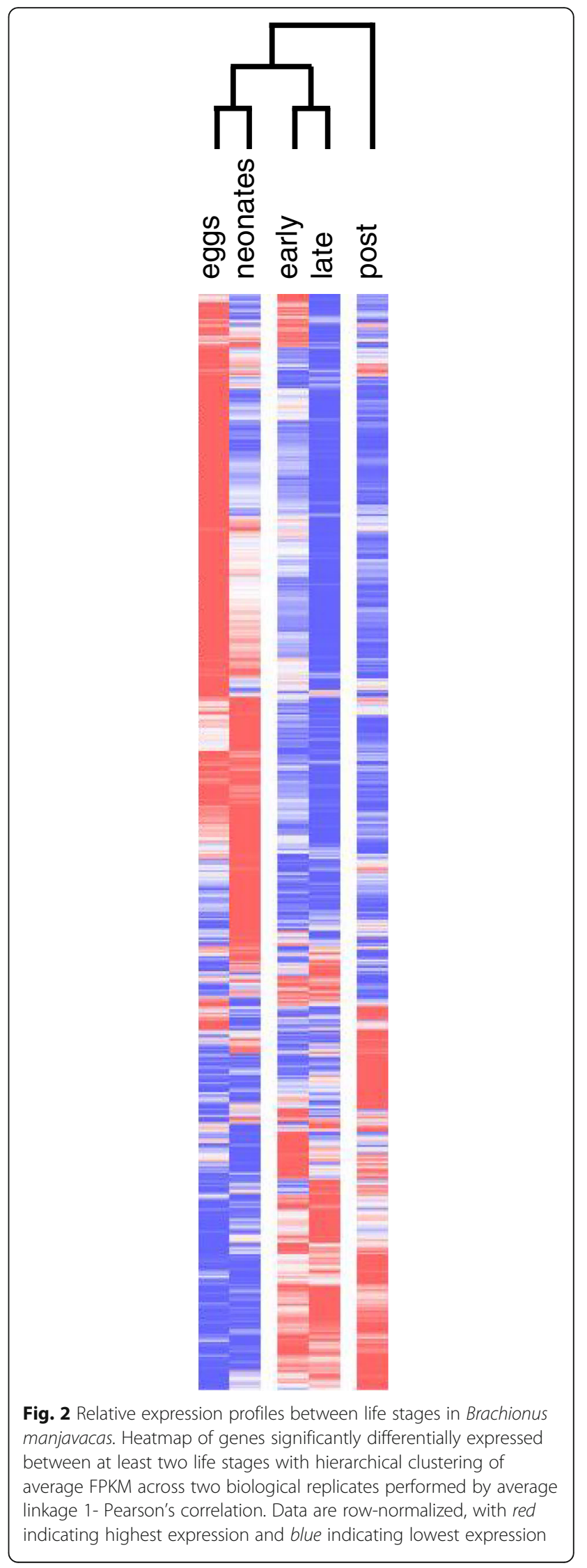




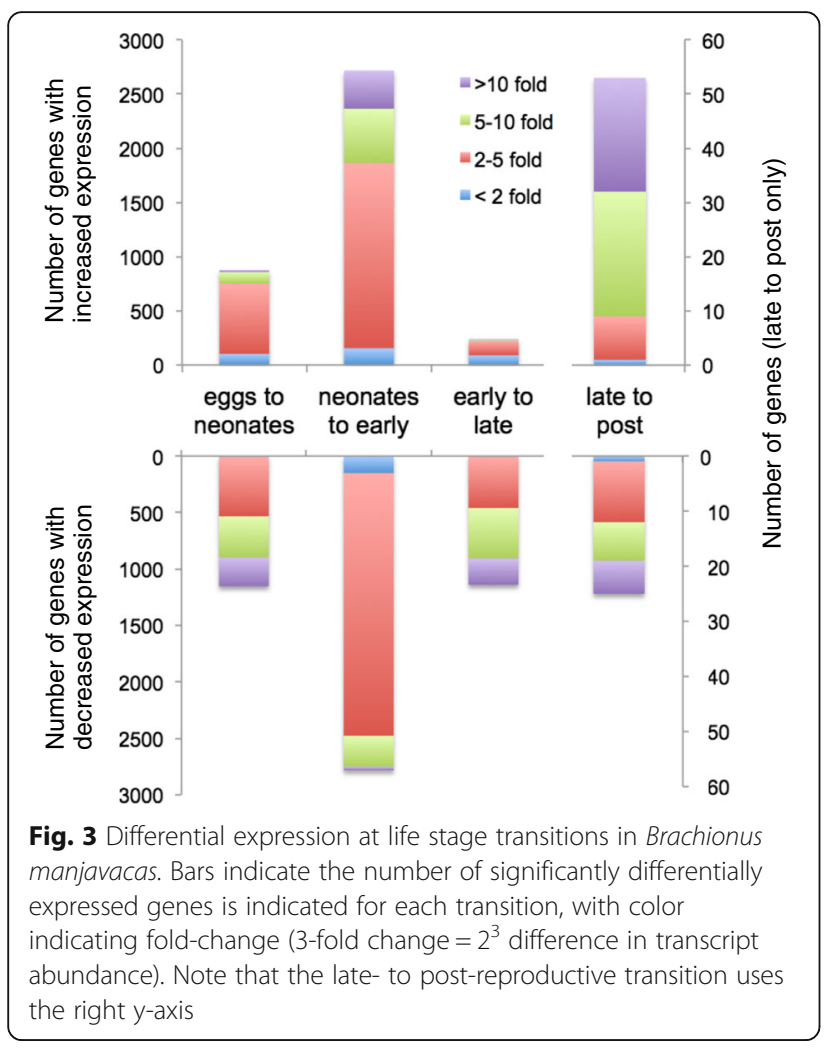

\section{Eggs to neonates}

Almost 10\% of the transcriptome (2028 genes) is differentially expressed in the transition from egg to neonate (Figs 2, 3 and 4). Enriched GO terms and KEGG pathways are characteristic of a switch from a developing embryo to a swimming, feeding animal.

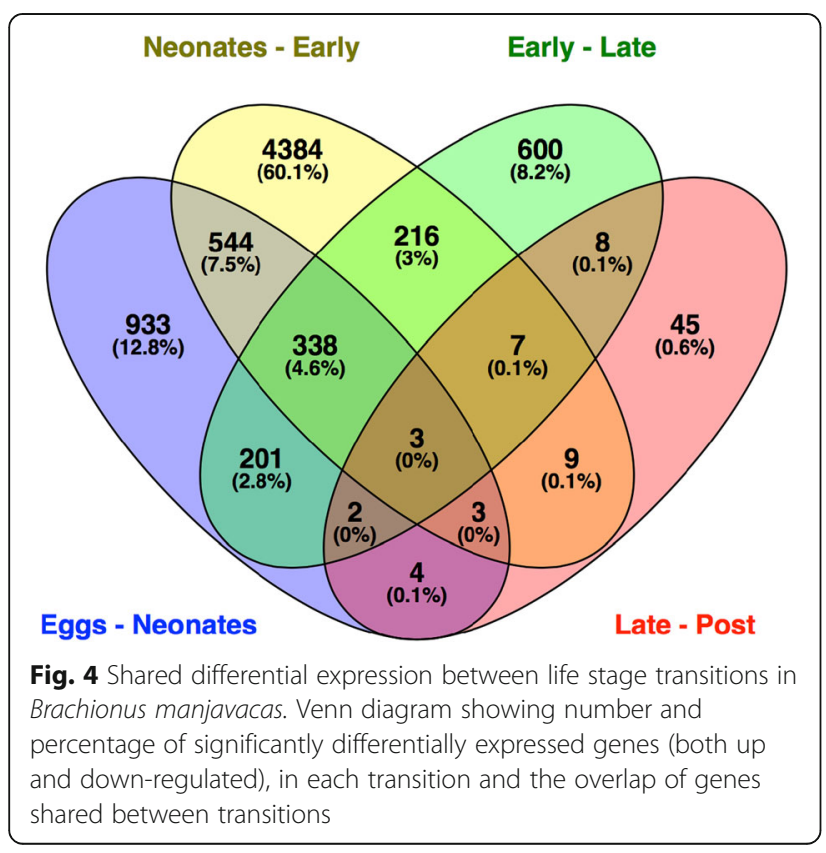

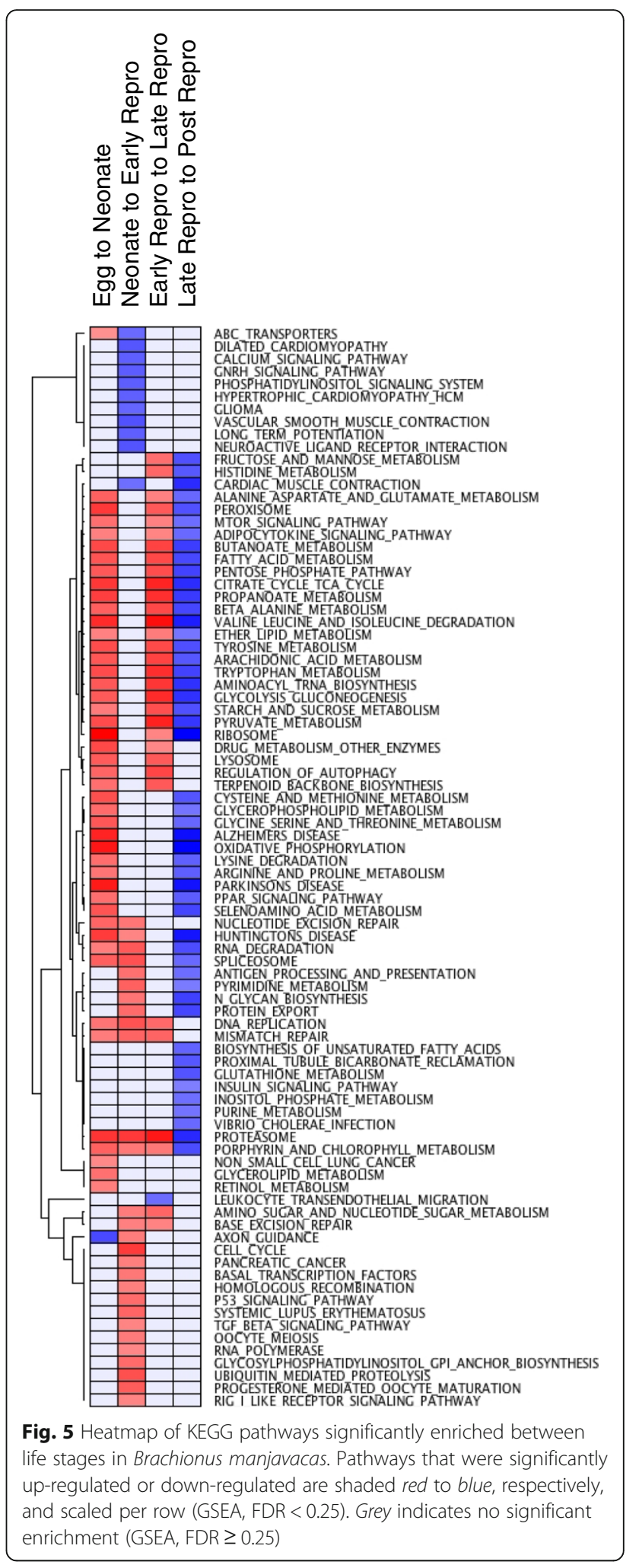

Nearly two thirds (715/1152) of genes that significantly decrease expression in this transition, many by more than $2^{5}$ (5 fold), do not increase expression at any later stage, indicating that they are highly expressed only in eggs and 
suggesting that they are specific to embryonic development. Consistent with this interpretation, these genes are involved in development, reflected in the down-regulation of GO terms including embryonic morphogenesis, organ morphogenesis, junction assembly, axon guidance, chemotaxis, and epithelium morphogenesis. The chitincontaining lorica and mastax of the rotifer are formed in the egg, and mastax size does not change after hatching, leading to the down-regulation of chitin catabolic processes after development (Additional file 2) [37].

Among all differentially expressed genes in this transition, $47 \%(413 / 876)$ of those that are up-regulated are later down-regulated, and 38\% (434/1152) of those that are down-regulated are up-regulated after the neonate stage, suggesting that these genes are specifically involved in development of the neonate. These genes are enriched in a relatively small number of GO terms and KEGG pathways, and are primarily integral membrane proteins including $\mathrm{ABC}$ transporters, solute carriers, and cytochrome P450s, as well as notch1, and phospholipase B1, glutathione synthetase, and alkaline phosphatase (Additional file 2, Fig. 5).

More than half of genes with increased expression $(455 / 876)$ do not decrease expression at any later stage and thus are specific to hatched animals rather than eggs. These genes are involved in metabolism, digestion, processing and excretion of waste, respiration, and sensing of the external environment.

\section{Neonates to early reproduction}

The transition from the neonate to early reproductive stage is accompanied by the greatest change in the transcriptome, with $25 \%$ (5504) of examined genes significantly up- or down-regulated and $30 \%$ of up-regulated genes increasing by more than 2-5 fold (Figs 2, 3 and 4). However, only 15\% of up-regulated genes (386/2718) and even fewer downregulated genes $(77 / 2786)$ are down-regulated or upregulated in later stages, respectively, indicating that relatively few of these genes are specific to early reproduction and are instead likely responsible for the transition from developmental to reproductive stages. Environmental interventions or genetic changes that modify expression of these genes may alter the timing of entry into adulthood and thus impact both healthspan and lifespan.

Pathways down-regulated in the transition from neonates to early reproduction include those involved in signaling and nervous system function, including $A B C$ transporters, GnRH signaling, long-term potentiation, and neuroactive ligand receptor interaction (Fig. 5). There is additional down-regulation of pathways involved in calcium-mediated muscle contraction and relaxation, including a decrease in expression of ATP2A2, tropomyosin and subunits of the troponin complex, actin, tintin, myosin light chain kinase, and voltage-dependent calcium channels. Other GO terms enriched in down-regulated genes include cell signaling and cell transport-neuro peptide Y receptor, G-protein coupled amine receptor, potassium channel, transmembrane transport and transport of organic anions, monamines, and sodium ions; macropinocytosis, an endocytic process, and monamine transport (Additional file 2). Taken together, these suggest developmental processes necessary for interaction with the environment, including gravitaxis, phagocytosis, and detection of abiotic or external stimuli, are completed during the neonatal stage.

The suite of genes up-regulated in the transition from neonates to early reproductive females appears to be driven largely by the onset of reproduction (Fig. 5, Additional file 2). Enriched pathways are dominated by the cell cycle (including positive and negative regulation of ubiquitinprotein ligase activity, cell cycle checkpoint, and regulation of mitosis); DNA replication (positive and negative regulation, leading and lagging stand elongation), recombination, and repair (ligase activity, DSB processing, homologous recombination, BER, NER, MMR); oogenesis; and maturation and cell division. Up-regulation of RNA polymerase, the spliceosome, and basal transcription factors suggest a general increase in transcription, while increases in cell cycle pathways, p53, and TGF-beta are expected to control cell growth, differentiation, apoptosis, and cellular homeostasis during growth and embryonic development.

\section{Early to late reproduction}

There are only 236 genes up-regulated in the transition to late reproduction; of these 104 are not differentially expressed in earlier transitions, and nearly all (224) are not differentially expressed in the transition to reproductive senescence (Figs 2, 3 and 4). Most of those pathways up-regulated in the early to late reproductive transition-primarily those involved in metabolism-were also up-regulated in either or both of the earlier transitions. An exception is carbohydrate metabolic processes, up-regulated only in this transition (Additional file 2). In contrast, 1140 genes are down-regulated, 504 of which are not differentially expressed at any other stage. GO terms associated with reproduction, development, cell migration and organ morphogenesis all declined (Additional file 2), but only the leukocyte transendothelial migration pathway was significantly down-regulated in GSEA (Fig. 5). This down-regulation was due to the decreased expression of Ras family genes, including ROCK1, RAC2, RAC1, RAP1B, and RHOA MYL9, ITK, and PRKCs. These genes are involved in cell growth, differentiation, and survival. As rotifers are eutelic, one would expect these cell division and differentiation genes to be down-regulated after development and with the decline in reproduction during this period. Decreases in subunits of cytochrome B-245, a component of the microbicidal oxidase system of 
phagocytes and of ITK may signal a decreased capacity to fight off infection with increasing age. Declines in the expression of MYL9, MYL12B, ACTB, ACTG and PRKCs suggest a breakdown in cell regulation.

\section{Late reproduction to post reproduction}

The transition to post-reproductive senescence has the smallest number of significantly differentially expressed transcripts (Figs 2, 3, and 4). Of the 53 upregulated and 25 down-regulated genes, 35 and nine, respectively, are not differentially expressed in any other transition. Of these, 21 transcripts are unannotated, and are targets for further investigation. Annotated transcripts are involved in aerobic respiration, signaling, and transport (Additional file 3).

In order to understand how more subtle changes in transcription level of individual genes could result in significant changes in genetic pathways, we focused on pathways identified as enriched by GSEA. Examining the core genes in pathways and whether they are differentially expressed in multiple related pathways can reveal genes likely to have a disproportionate impact on aging.

Consistent with clustering of transcriptional profiles (Fig. 2) we identified multiple pathways differentially expressed only in the late- to post-reproductive transition. Pathways down-regulated in this transition but not enriched in any other include biosynthesis of unsaturated fatty acids, tubule reclamation, glutathione metabolism, insulin signaling, inositol phosphate metabolism, and purine metabolism (Fig. 5).

Pathways up regulated in one or more of the first three transitions and down-regulated in the transition to reproductive senescence were primarily in metabolism, maintenance and repair, and proteostasis (Fig. 5). Many of these seemingly distinct pathways, including those implicated in the age-related diseases of Parkinson's, Huntington's, and Alzheimer's, were united by genes involved in mitochondrial function and oxidative phosphorylation, including NDUFA, UQCRC, COX genes, CYC1, SDHA, VDAC2 and SLC25A31, all components of the respiratory chain (Fig. 6). Declines in expression in these same pathways with aging appear to be evolutionarily conserved across a range of taxa $[1,38-40]$.

\section{Metabolism}

GSEA revealed a suite of metabolic pathways downregulated in aged rotifers, including fatty acid, pentose phosphate, porphyrin and ether lipid metabolism, glycolysis, and the TCA cycle (Fig. 5). Multiple dehydrogenases were among the genes with the largest decreases in expression, including aldehyde dehydrogenases, hydroxyacyl-CoA dehydrogenase (HADH), malate dehydrogenases (MDH2, MDH1), and pyruvate dehydrogenase (PDHA1, PDHB). Phospholypases (PLAG2E, PLA2G3), aldolases (ALDOA,

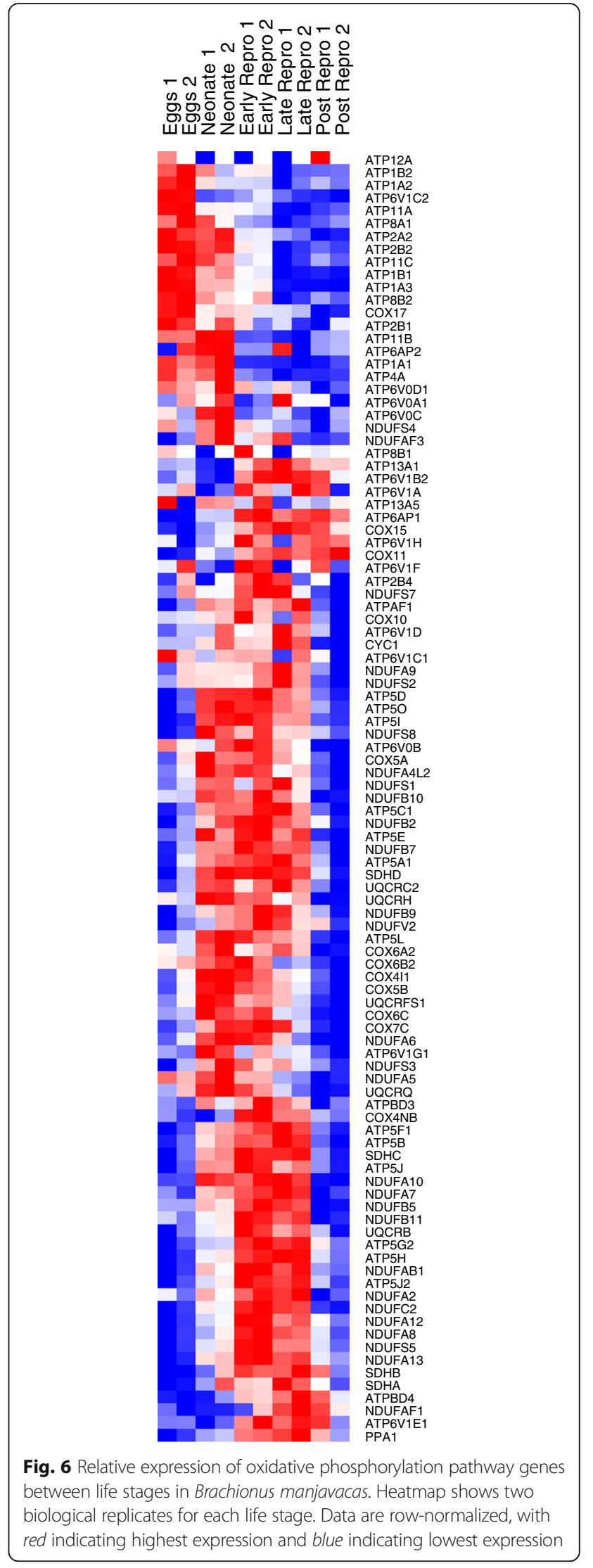


ALDOC), phosphoenolpyruvate carboxykinase 1 (PCK1), and pyruvate kinase (PKM2) also all decreased in the transition from late- to post reproduction (Additional file 4).

Important signaling pathways with roles in metabolism, nutrient sensing, and oxidative phosphorylation were also down-regulated in post reproduction, most notably insulin, tor, and adipocytokine signaling. These pathways are known to play an important role in aging, and are effectors in increases in longevity in response to metabolism-related therapies, including caloric restriction [38, 41]. Expression of key agingrelated genes, including JNK, INSR, IGF, IGFALS, and TOR-RNAi knockdown of which is known to increase lifespan in rotifers and other animal models $[25,42-45]$-all increase slightly in senescence (Fig. 7). Other components of the insulin signaling and TOR pathways, particularly calcium-signaling genes, exhibit decreases in expression late in life. Together, these data suggest a synchronized breakdown in metabolic function and capacity late in life.

\section{Proteostasis}

A decline in protein homeostasis is considered one of the hallmarks of aging across taxa [1], and our results suggest such a decline in late life in B. manjavacas. Expression of nearly all proteasome-related genes increases steadily over life until the transition to reproductive senescence, when expression of 31 out of 38 annotated structural proteasome subunit and catalytic co-factor genes decreased by up to 2.4-fold (Fig. 8); GSEA results capture the late-life decline in proteasome expression (Fig. 5). The proteasome degrades damaged or shortlived endogenous proteins, particularly cyclins and transcription factors, and recycles amino acids to be used in synthesis of new proteins. Proteasome function is thus essential for many cellular processes, including the regulation of gene expression, the cell cycle, and the response to cellular stress. A decline in the proteasome leads to an accumulation of mis-folded and aggregated proteins resulting in proteotoxic stress. A loss in proteostasis, aggregation of proteins, and widespread changes in the proteome are associated with aging in $C$. elegans $[46,47]$. Interestingly, expression of the ubiquinated proteolysis pathway, which targets damaged proteins for removal via the proteasome, did not change significantly over lifespan in rotifers, suggesting that globally, ubiquitination is not the rate-limiting step in proteasomal degradation in late age, though declines in ubiquitination of specific targets likely play a role in aging.

Protein metabolism pathways were generally downregulated in post-reproductive rotifers (Fig. 5). Leading edge analysis revealed the subset of genes that were commonly down-regulated between multiple protein metabolism

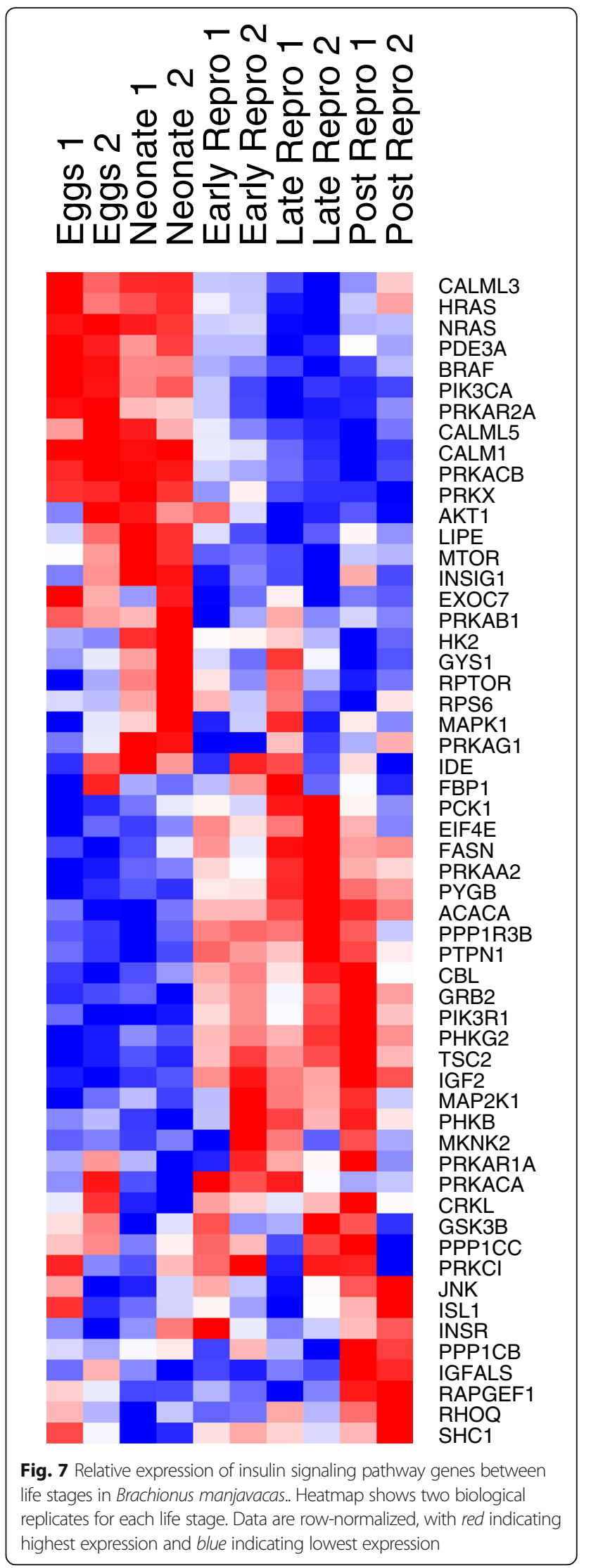




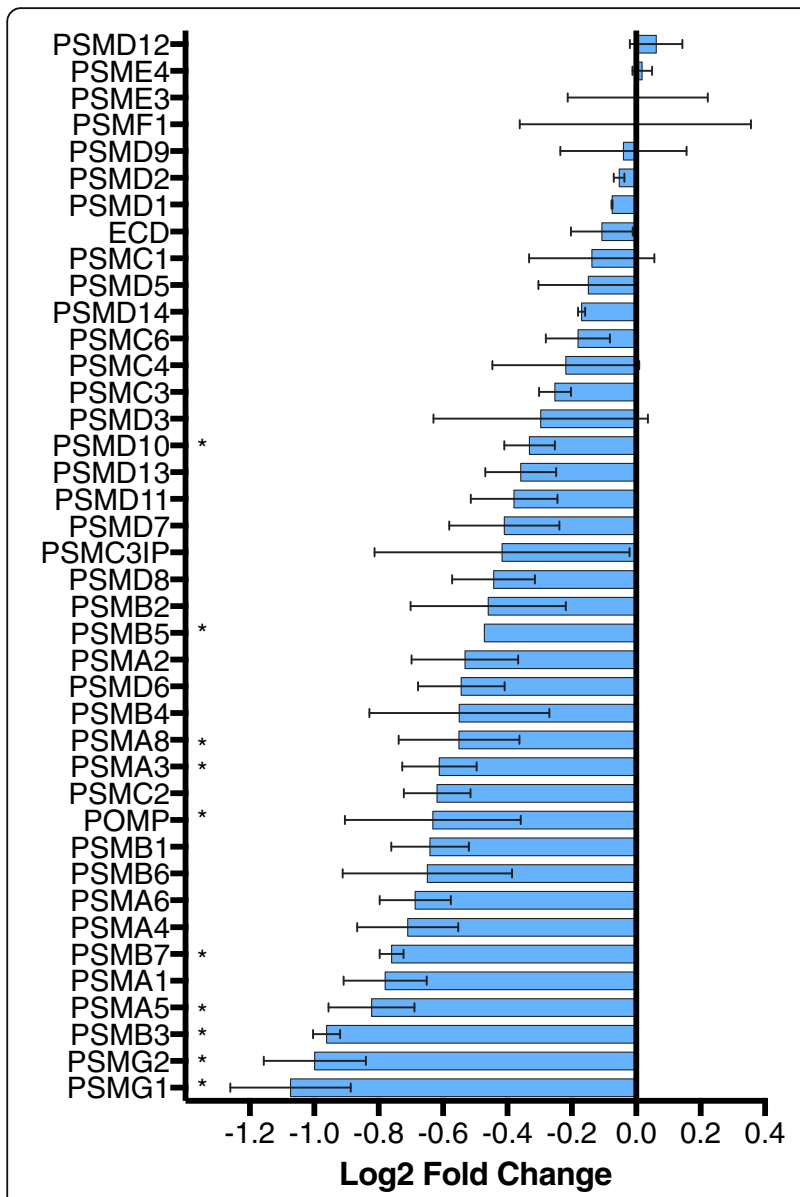

Fig. 8 Expression change of proteasome subunit and assembly genes in the late- to post reproduction transition. Bars show mean fold change \pm standard deviation of two biological replicates. * indicates significantly different expression between the two life stages ( $p \leq 0.05$, one-tailed $t$-test)

pathways (Additional file 4). These were generally involved in catalyzing formation and then breakdown of $\mathrm{S}$ adenosylmethionine (MAT1A, MAT2A, AHCY, CTH, and WBSCR22), ultimately decreasing the production of methyl groups available for methylation. Expression of the protein methyltransferases METTL6, METTL2B, and LCMT2 also declined. A suite of dehydrogenase enzymes that function in the mitochondria were down-regulated (GCDH, ALDH3A2, ALDH2, HADH, HADHA, ALDH4A1, GLUD1), affecting most protein metabolism pathways and linking amino acid metabolism and degradation with the butanoate and propanoate metabolism pathways.

Expression of all the enzymes involved in the highly conserved, five-step tyrosine degradation pathway declined in post-reproductive rotifers (Fig. 9). Tyrosine is an important regulator of larval development and adult longevity in C. elegans, and acts as a signaling molecule involved in cell differentiation, growth, and maintenance [48]. Expression of homogentisate 1,2-dioxygenase (HGD)

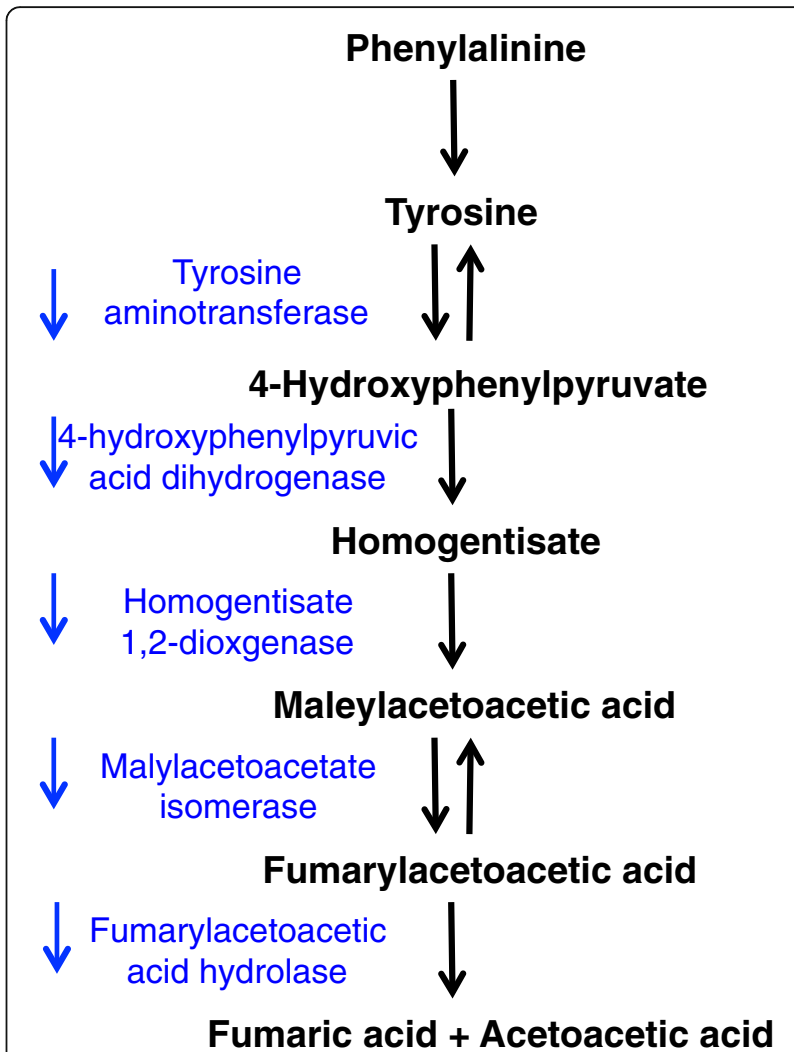

Fig. 9 Expression change of tyrosine metabolism pathway genes between late- and post reproduction in Brachionus manjavacas. Blue arrows indicate the observed decline in expression of enzymes that catalyze metabolism of tyrosine

doubles in the transition from eggs to neonates, decreases slightly in the transition to early reproduction, doubles again in late reproduction, and declines again to less than half of previous levels in post-reproductive females. HGD is involved in the catabolism of tyrosine and phenylalanine and is best known as the autosomal recessive cause of alkaptonuria when mutated. Decreased function of HGD leads to a damaging buildup of homogentisic acid in connective tissues, and the inability to recycle phenylalanine and tyrosine into new proteins [49]. Increases in tyrosine concentration or changes in post-translational modification are associated with age-related disease including cancer, diabetes, neurodegeneration, and cataracts $[48,50]$. Tyrosine metabolism is tied with metabolism and signaling, and is actively controlled by insulin signaling and in turn tyrosine aminotransferase (TAT), which catalyzes the conversion of tyrosine to fumarate and acetoacetate, appears to modify the effects of daf2/IGFR through FOXO and AMPK, thus strongly tying metabolic and protein sensing, signaling, and regulation.

Cathepsin C (CC; dipeptidyl peptidase-1) expression significantly increased 2.2 fold in senescent rotifers (baySeq, $p<0.05)$. CC is a lysosomal cysteine protease that activates 
granule serine peptidases in inflammatory cells. Increases in CC are associated with inflammatory diseases, and CC protein levels are increased in the central nervous system in aging mice, contributing to pathogenic inflammation [51]. Across taxa, aging is correlated with chronic inflammation that is associated with disease, decline in physical function, and mortality; this remains an active area of investigation in the biology of aging $[52,53]$.

\section{Signaling and interaction with the environment}

The calcium pathway shows a dramatic shift over lifespan in rotifers, with the highest expression across nearly all calcium pathway related genes in eggs and neonates, a dramatic decrease during early and late reproduction, then a slight rise in expression of a subset of genes in the postreproductive period (Fig. 10, Additional file 5). Several kinases involved in calcium signaling, including PhKG2, PRKACA and ITPKB also increase in late life. In contrast, there is decreased expression of SERCA (ATP2A2) calcium pumps in the endoplasmic reticulum, which could decrease cellular calcium buffering capacity, as found in aged neurons [54]. In rotifers, as in other animals, mitochondria appear to have a decreased ability to take up calcium in late life $[55,56]$.

The overall effect of the observed changes in gene expression is likely to be dysregulation of calcium homeostasis in senescent rotifers, leading to an increased intracellular calcium load that may negatively impact neuronal excitability [57-59]. In other model systems, loss of calcium homeostasis has been shown to be common to several age-related neurodegenerative diseases, including Alzheimers, Parkinson's, and ALS [54, 60, 61]. The dysregulation of signaling and of sensing pathways, including neuron recognition and chemotaxis, beginning as soon as the early-reproductive period should be investigated further. Loss of the ability to sense and react appropriately to environmental conditions would be detrimental in late life.

\section{Epigenetic control of transcription across all life stages}

The level of 5-methylcytosine in Brachionus is below reliable limits of detection by LC-MS [62], and we and others have not found DNA methyltransferases (Dnmt1, Dnmt3) in the B. manjavacas transcriptome or in other published rotifer transcriptomes or genomes [15, 31, 62]. However, we report here that $B$. manjavacas has the molecular machinery for post-translational modifications to histone tails, including histone methylation and acetylation; these modifications play an important role in regulating gene expression. Most histone acetylases (HAT1, KAT5, KAT7, KAT8, CLOCK, ELP1) remained relatively constant with age, while histone deacetylases, activity of which is associated with repressed transcription, increased after the developmental period in eggs

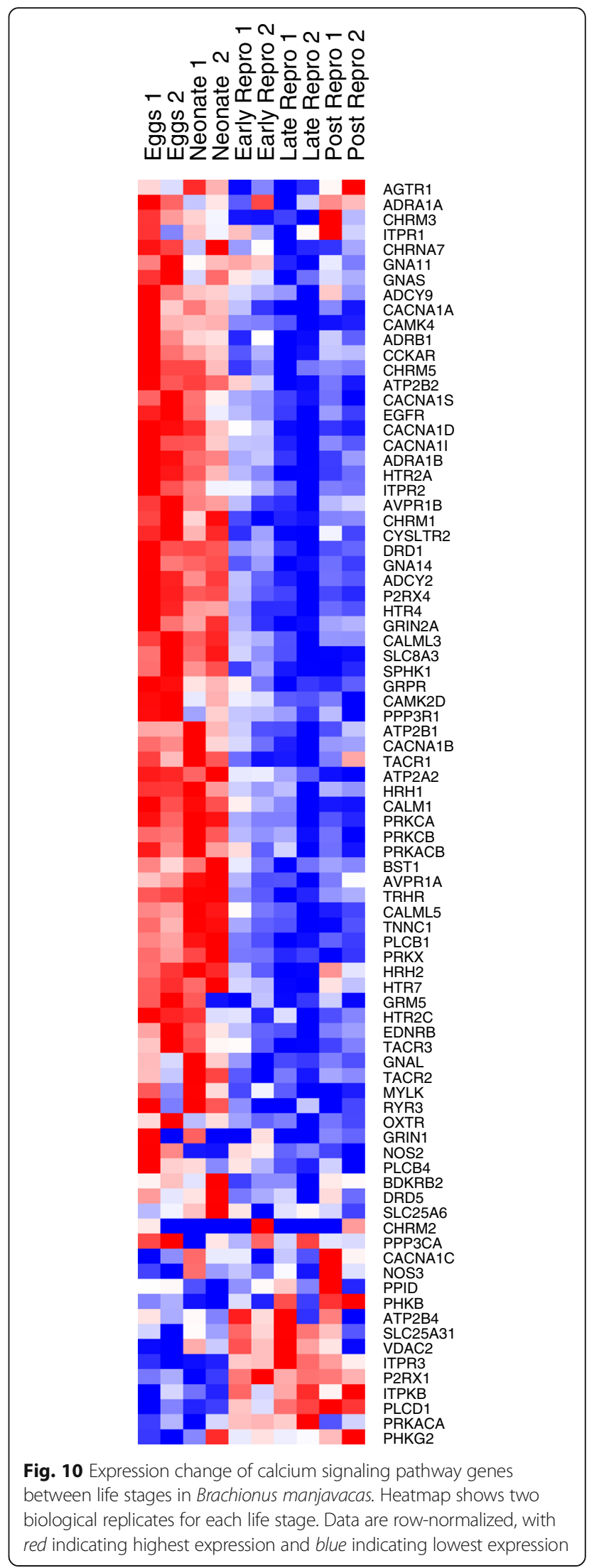




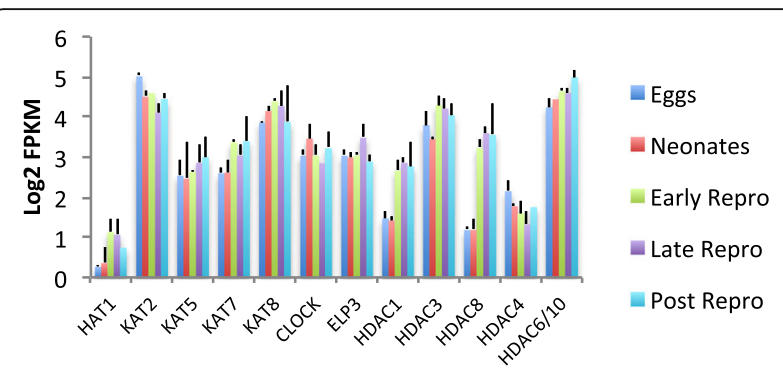

Fig. 11 Expression of histone acetylase and deacetylase genes at each life stage in Brachionus manjavacas. Bars show mean expression as log2 of Fragments Per Kilobase of transcript per Million mapped reads $($ FPKM $) \pm$ standard deviation of two biological replicates

and neonates and remained high through late life (Fig. 11). Expression of HDAC6/10 was highest in postreproductive rotifers. Only HDAC4 declined slightly but steadily from eggs to late reproduction.

Sirtuin proteins are NAD+ dependent deacetylases, and are conserved regulators of aging and age-related diseases [63]. In B. manjavacas we found homologs of human sirtuins, based on amino acid sequence similarity: SIRT1 (nuclear), SIRT2 (cytoplasmic), SIRT3, SIRT4 (both mitochondrial) and SIRT7 (nuclear). Expression of SIRT1 and SIRT3 were relatively constant over rotifer lifespan (Fig. 12). Decrease in SIRT1 (the mammalian ortholog of yeast Sir2) by RNAi shortens lifespan in model organisms, while lifespan-extending caloric restriction up-regulates SIRT1. While SIRT4 expression was low, it rose 1.9-fold over the transitions from eggs to neonates to early reproduction. Sirt4 is a mitochondrial protein with additional activity as an NADdependent protein ADP-ribosyl transferase. SIRT2 expression, conversely, is high through development and early life, then declines by 1.1-fold through the reproductive and post-reproductive period. Sirt2 deacetylates

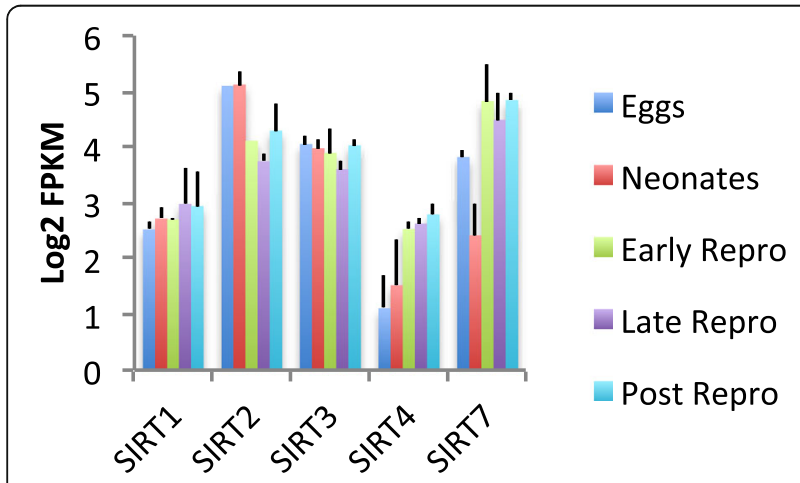

Fig. 12 Expression of sirtuin family genes at each life stages in Brachionus manjavacas. Bars show mean expression as log2 of Fragments Per Kilobase of transcript per Million mapped reads $($ FPKM $) \pm$ standard deviation of two biological replicates a number of substrates, including H3K56, and H3K16, FOXO3, and alpha-tubulin [64-66]. Expression of SIRT7 displays the opposite profile, and is low in eggs and neonates then rises by 2.7 -fold to remain high for the rest of life. SIRT7 specifically mediates deacetylation of H3K18, and is directly linked to the control of gene expression, particularly of nuclear hormone receptors [67]. SIRT7 plays a role in oncogenic transformation by suppressing the expression of tumor suppressor genes, and in humans SIRT7 expression is significantly elevated in breast cancer and thyroid carcinoma $[68,69]$.

Histone methyltransferases demonstrated a shift in expression between the first two (developmental) and last three (early-, late-, and post-reproductive) life stages (Fig. 13). In particular, expression of several genes that methylate H3K4, causing transcriptional activation, increased after development (KMT2A, 1.8-fold increase, KMT2B; 1.5-fold increase; SETD1B, 1.4-fold increase). Other, repressive histone methyltransferases also increased expression slightly in late life, including SETD8, SETD1B, EHMT, while expression of SUV420H1 decreased. The repressive polycomb group protein $\mathrm{EZH}$, which methylates $\mathrm{H} 3 \mathrm{~K} 9$ and $\mathrm{H} 3 \mathrm{~K} 27$ and plays a key role in development and differentiation [70], declined in expression by 1.4-fold from neonates to early reproduction, and another 1.0-fold from early- to late reproduction.

There was little to no up-regulation of histone lysine demethylases in late age; only KDM1A, which demethylates $\mathrm{H} 3 \mathrm{~K} 4$ and $\mathrm{H} 3 \mathrm{~K} 9$, repressing transcription, increased 0.4-fold from early- to late reproduction, then declined by the same amount from late- to post reproduction. Expression of the H3R2 and H4R3 demethylase JMJD6 increased 1.1-fold between neonates and early reproduction, resulting in repression of transcription.

Together, these results suggest that large shifts in epigenetic markers may be partly or even largely responsible for driving the changes in gene expression over lifespan, an idea increasingly supported by results from other model systems [71, 72]. SETD1 and SETMAR provide particularly interesting targets for future investigation, as their expression changes greatly over lifespan, and not only in the transition from development to growth and reproduction. The increase (0.8-fold) of SETMAR and large decrease in SETD1A (5.2-fold) in the transition from late- to post reproduction suggests that they have functions beyond development, cell division, and differentiation.

\section{Differential expression during aging revealed in the rotifer model}

Our assembly of the $B$. manjavacas transcriptome included 614 and 910 transcripts with potential homologs in the human genome absent in D. melanogaster and $C$. elegans genomes, respectively. Of these, 150 and 262 

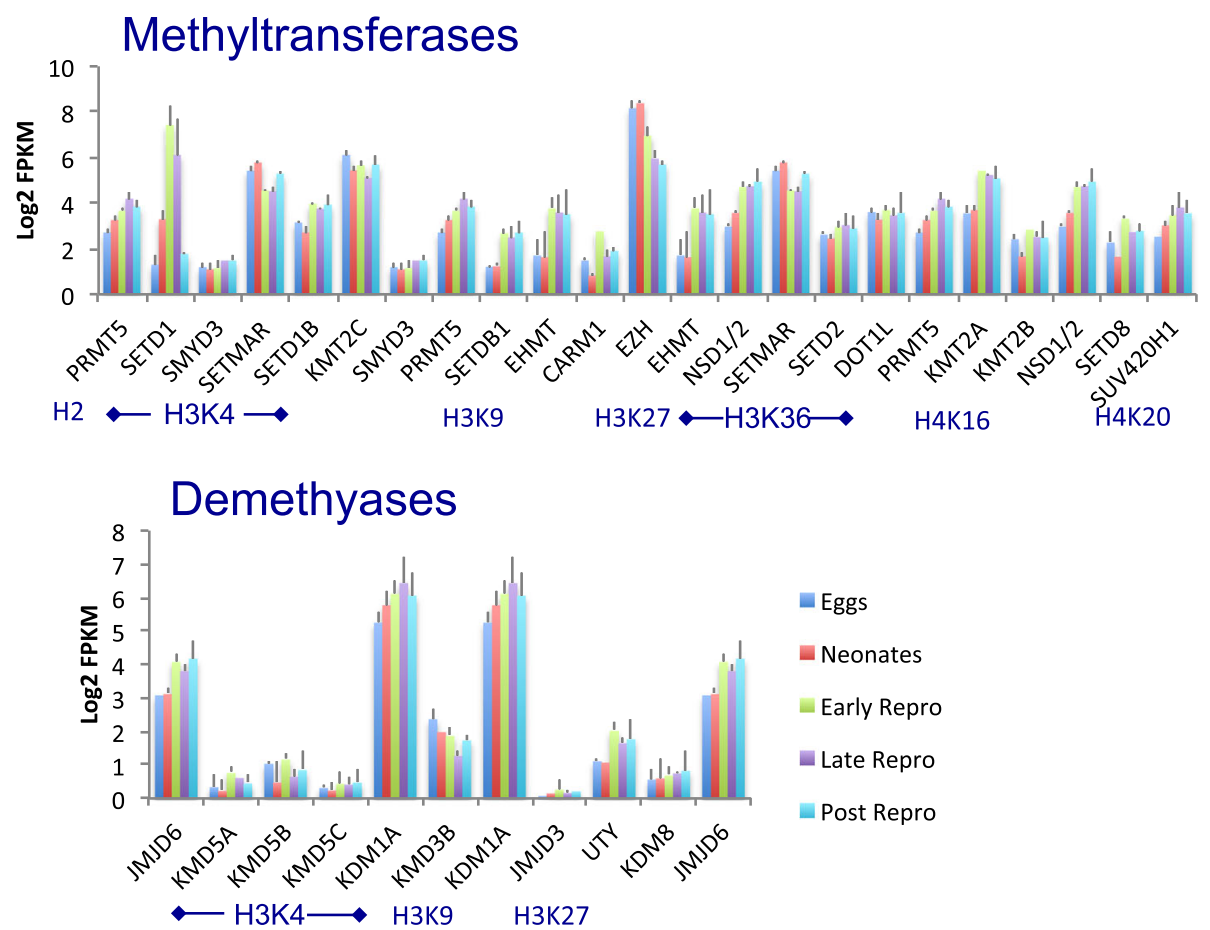

Fig. 13 Expression of histone methyltransferase and demethylase genes at each life stages in Brachionus manjavacas. Bars show mean expression as log2 of Fragments Per Kilobase of transcript per Million mapped reads (FPKM) \pm standard deviation of two biological replicates

$(\sim 25 \%)$ were significantly differentially expressed in at least one life stage transition. Differentially expressed transcripts that lack homologs in both flies and worms correspond to 93 human genes, of which 20 are differentially expressed between eggs and neonates, 69 between neonates and early reproduction, 10 between early and late reproduction, and one between late reproduction and senescence (Additional file 6). In the egg to neonate transition, increased expression of arsenite methyltransferase (AS3MT), methionine adenosyltransferase 2B (MAT2B), and thiopurine S-methyltransferase (TPMT) enrich GO terms related to xenobiotic stimulus. In the neonate to early reproductive transition, increased expression of 12 genes enriched GO terms related to the cell cycle and DNA metabolic processes. Five of these and an additional three genes enrich GO terms associated with the microtubule organizing center and centrosome. The centrosome is involved in chromosome stability and has been suggested to play a role in aging [73-75].

\section{Conclusions}

This study is the first to provide a detailed examination of the transcriptome and of changes in gene expression and gene pathways with normal aging in the rotifer Brachionus manjavacas, an emerging animal model for the study of the biology of aging. Shifts in expression in major aging related pathways very early in life suggest that changes in gene expression that cause or are caused by aging may actually begin at a relatively young age. We found a general decline in metabolism, signaling, proteostasis, and mitochondrial function associated with aging. Our findings highlight the important role in aging of changes in pathways that integrate many diverse functions, and suggest that subtle changes in expression of many genes, rather than a dramatic change in expression of individual genes, may lead to significant shifts in the function of biochemical pathways. A decline in pathways involved in cellular maintenance and repair such as the proteasome will lead to the accumulation of damage and prevent the recycling of cellular components. Signaling pathways such as calcium and insulin exert control across cellular and organismal processes as disparate as development, metabolism and neural function. The simultaneous declines in expression of energetic, signaling, and amino acid metabolism pathways are likely to have very different consequences for health and longevity than single or multi-gene knockdown in an otherwise healthy animal; thus investigation of subtle but genomewide change in amino acid sensing and metabolism pathways during aging is an important area for future study. These results offer a framework that may be used to generate new hypotheses about the molecular genetic mechanisms of aging and provide a baseline against which to evaluate changes in gene expression due to interventions that change healthspan and lifespan. 


\section{Additional files}

Additional file 1: $\mathrm{R}$ script used for running baySeq. (TXT $4 \mathrm{~kb}$ )

Additional file 2: Gene Ontology terms significantly differentially regulated in sequential life history transitions (Blast2GO, FDR < 0.05) Categories: $\mathrm{C}=$ cellular component, $\mathrm{F}=$ molecular function, $\mathrm{P}=$ biological process. Worksheet 1: GO terms significantly up-regulated in neonates relative to eggs; Worksheet 2 . GO terms significantly down-regulated in neonates relative to eggs; Worksheet 3: GO terms significantly upregulated in early reproduction relative to neonates; Worksheet 4: GO terms significantly down-regulated in early reproduction relative to neonates; Worksheet 5: GO terms significantly up-regulated in late reproduction relative to early reproduction; Worksheet 6: GO terms significantly down-regulated in late reproduction relative to early reproduction. (XLSX $53 \mathrm{~kb}$ )

Additional file 3: Table of genes significantly differentially expressed in the late-to post-reproductive transition and at least one other transition, with KEGG description, and with charts showing changes in expression in genes significantly up (top) or down (bottom) regulated in the late- to post-reproductive transition. Forty-one unannotated genes were also upregulated, and eight unannotated genes were down-regulated. (DOCX 143 kb)

Additional file 4: Leading edge analysis results for protein, carbohydrate, and fatty acid metabolism pathways. Worksheet 1: Change in gene expression in multiple protein metabolism pathways, as determined by leading edge analysis in GSEA. Shows those genes that are shared in more than one protein metabolism pathway (as denoted by X), not all genes in all pathways; Worksheet 2: Change in gene expression in multiple carbohydrate and fatty acid metabolism pathways, as determined by leading edge analysis in GSEA. Shows those genes that are shared in more than one protein metabolism pathway (as denoted by $\mathrm{X}$, not all genes in all pathways. (XLSX $48 \mathrm{~kb}$ )

Additional file 5: The KEGG calcium signaling pathway showing genes up-regulated (red) and down-regulated (blue) in the late- to postreproductive transition. (PNG $172 \mathrm{~kb}$ )

Additional file 6: Transcripts with potential orthologs in humans and not in D. melanogaster or C. elegans. (TXT $44 \mathrm{~kb}$ )

\section{Abbreviations}

FPKM: Fragments mapped per kilobase of transcript per million reads; GO: Gene ontology; GSEA: Gene set enrichment analysis; HGNC: HUGO gene nomenclature committee; HUGO: Human genome organization

\section{Acknowledgements}

We thank Terry Snell for cultures, Bette Hecox-Lea and Joe Vineis for library preparation and sequencing, Nicola Neretti for assistance with GSEA, and two anonymous reviewers for their thoughtful comments on the manuscript.

\section{Funding}

Funding for this project was provided by R01 AG037960-01, the American Federation for Aging Research, and the Bay and Paul Foundations.

\section{Availability of data and materials}

The datasets generated and analyzed during the current study are available in the NCBI BioProjects PRJNA345262 (raw reads used in mapping) and PRJNA368952 (assembled B. manjavacas transcriptome).

\section{Authors' contributions}

KEG performed the experiments. Both authors designed the experiments, analyzed the results, and wrote the manuscript. Both authors approved the final manuscript.

\section{Competing interests}

The authors declare that they have no competing interests.

\section{Consent for publication}

Not applicable.
Ethics approval and consent to participate

Not applicable.

Received: 28 September 2016 Accepted: 2 February 2017

Published online: 01 March 2017

\section{References}

1. López-Otín C, Blasco MA, Partridge L, Serrano M, Kroemer G. The hallmarks of aging. Cell. 2013;153:1194-217.

2. Takahashi T, McDougal C, Troscianko J, Chen W-C, Jayaraman-Nagarajan A, Shimeld SM, Ferrier DEK. An EST screen from the annelid Pomatoceros lamarckii reveals patterns of gene loss and gain in animals. BMC Evol Biol. 2009;9:240.

3. Wyder S, Kriventseva E, Schroder R, Kadowaki T, Zdobnov E. Quantification of ortholog losses in insects and vertebrates. Genome Biol. 2007;8(11):R242.

4. Zmasek CM, Zhang Q, Ye Y, Godzik A. Surprising complexity of the ancestral apoptosis network. Genome Biol. 2007;8(10):R226.

5. Dieterich C, Clifton SW, Schuster LN, Chinwalla A, Delehaunty K, Dinkelacker I, Fulton L, Fulton R, Godfrey J, Minx P, et al. The Pristionchus pacificus genome provides a unique perspective on nematode lifestyle and parasitism. Nat Genet. 2008:40(10):1193-8.

6. Jiang $\mathrm{L}, \mathrm{Ni} J$, Liu Q. Evolution of selenoproteins in the metazoan. BMC Genomics. 2012;13:446

7. Kortschak RD, Samuel G, Saint R, Miller DJ. EST analysis of the cnidarian Acropora millepora reveals extensive gene loss and rapid sequence divergence in the model invertebrates. Curr Biol. 2003:13(24):2190-5.

8. Matsui T, Yamamoto T, Wyder S, Zdobnov EM, Kadowaki T. Expression profiles of urbilaterian genes uniquely shared between honey bee and vertebrates. BMC Genomics. 2009;10:17.

9. Philipp EER, Kraemer L, Melzner F, Poustka AJ, Thieme S, Findeisen U, Schreiber S, SRosenstiel P. Massively parallel RNA sequencing identifies a complex immune gene repertoire in the lophotrochozoan Mytilus edulis. PLoS One. 2012;7(3):e33091.

10. Edgecombe G, Giribet G, Dunn C, Hejnol A, Kristensen R, Neves R, Rouse G, Worsaae K, Sørensen M. Higher-level metazoan relationships: recent progress and remaining questions. Org Divers Evol. 2011;11(2):151-72.

11. Dunn CW, Hejnol A, Matus DQ, Pang K, Browne WE, Smith SA, Seaver E, Rouse GW, Obst M, Edgecombe GD, et al. Broad phylogenomic sampling improves resolution of the animal tree of life. Nature. 2008:452(7188):745-9.

12. Austad SN. Is there a role for new invertebrate models for aging research? J Gerontol. 2009:64A(2):192-4.

13. Enesco HE. Rotifers in aging research; use of rotifers to test various theories of aging. Hydrobiologia. 1993:255/256:59-70.

14. Snell TW, Johnston RK, Gribble KE, Mark Welch DB. Rotifers as experimental tools to investigate aging. Invertebr Reprod Dev. 2015;59(1):5-10.

15. Flot J-F, Hespeels B, Li X, Noel B, Arkhipova I, Danchin EGJ, Hejnol A Henrissat B, Koszul R, Aury J-M, et al. Genomic evidence for ameiotic evolution in the bdelloid rotifer Adineta vaga. Nature. 2013;500(7463):453-7.

16. Hanson SJ, Stelzer C-P, Mark Welch DB, Logsdon J, John M. Comparative transcriptome analysis of obligately asexual and cyclically sexual rotifers reveals genes with putative functions in sexual reproduction, dormancy, and asexual egg production. BMC Genomics. 2013;14:412.

17. Wallace RL. Rotifers: exquisite metazoans. Integr Comp Biol. 2002;42:660-7.

18. Snell TW, Fields AM, Johnston RK. Antioxidants can extend lifespan of Brachionus manjavacas (Rotifera), but only in a few combinations. Biogerontology. 2012;13(3):261-75.

19. Ozaki Y, Kaneko G, Yanagawa Y, Watabe S. Calorie restriction in the rotifer Brachionus plicatilis enhances hypoxia tolerance in association with the increased mRNA levels of glycolytic enzymes. Hydrobiologia. 2010;649:267-77.

20. Tanaka C, Hashimoto Y, Nakao S, Yoshinaga T. Effect of juglone on the survival time of two Brachionus species (Rotifera): species-specific tolerance against oxidative stress. Fish Sci. 2009;75:191-4.

21. Kailasam M, Kaneko G, Oo AKS, Ozaki Y, Thirunavukkarasu AR, Watabe S. Effects of calorie restriction on the expression of manganese superoxide dismutase and catalase under oxidative stress conditions in the rotifer Brachionus plicatilis. Fish Sci. 2011;77:403-9.

22. Johnston RK, Snell TW. Moderately lower temperatures greatly extend the lifespan of Brachionus manjavacas (Rotifera): Thermodynamics or gene regulation? Exp Gerontol. 2016;78:12-22. 
23. Kaneko G, Yoshinaga T, Yanagawa Y, Ozaki Y, Tsukamoto K, Watabe S. Calorie restriction-induced maternal longevity is transmitted to their daughters in a rotifer. Funct Ecol. 2011;25(1):209-16.

24. Oo AKS, Kaneko G, Hirayama M, Kinoshita S, Watabe S. Identification of genes differentially expressed by calorie restriction in the rotifer (Brachionus plicatilis). J Comp Physiol B. 2010;180:105-16

25. Snell TW, Johnston RK, Rabeneck B, Zipperer C, Teat S. Joint inhibition of TOR and JNK pathways interacts to extend the lifespan of Brachionus manjavacas (Rotifera). Exp Gerontol. 2014;52:55-69.

26. Gribble KE, Jarvis G, Bock MJ, Mark Welch DB. Maternal caloric restriction partially rescues the deleterious effects of advanced maternal age on offspring. Aging Cell. 2014;13(4):623-30.

27. Gribble KE, Kaido O, Jarvis G, Mark Welch DB. Patterns of intraspecific variability in the response to caloric restriction. Exp Gerontol. 2014;51:28-37.

28. Gribble KE, Mark Welch DB. Life-span extension by caloric restriction is determined by type and level of food reduction and by reproductive mode in Brachionus manjavacas (Rotifera). J Gerontol Ser A Biol Med Sci. 2013; 68(4):349-58.

29. Guillard RRL. Culture of phytoplankton for feeding marine invertebrates. In: Smith WL, Chanley MH, editors. Culture of marine invertebrates. New York: Plenum Publishing Corporation; 1975.

30. Minoche AE, Dohm JC, Himmelbauer H. Evaluation of genomic highthroughput sequencing data generated on illumina HiSeq and genome analyzer systems. Genome Biol. 2011;12:R112.

31. Wey-Fabrizius AR, Herlyn H, Rieger B, Rosenkranz D, Witek A, Mark Welch DB, Ebersberger I, Hankeln T. Transcriptome data reveal Syndermatan relationships and suggest the evolution of endoparasitism in Acanthocephala via an epizoic stage. PLoS One. 2014;9(2):e88618.

32. Hardcastle T, Kelly K. BaySeq: empirical Bayesian methods for identifying differential expression in sequence count data. BMC Bioinf. 2010;11(1):422.

33. Mootha VK, Lindgren CM, Eriksson K-F, Subramanian A, Sihag S, Lehar J, Puigserver P, Carlsson E, Ridderstrale M, Laurila E, et al. PGC-1 [alpha]responsive genes involved in oxidative phosphorylation are coordinately downregulated in human diabetes. Nat Genet. 2003;34(3):267-73.

34. Subramanian A, Tamayo P, Mootha VK, Mukherjee S, Ebert BL, Gillette MA, Paulovich A, Pomeroy SL, Golub TR, Lander ES, et al. Gene set enrichment analysis: a knowledge-based approach for interpreting genome-wide expression profiles. Proc Natl Acad Sci. 2005;102(43):15545-50.

35. Thomas M, Yang L, Carter B, Klaper R. Gene set enrichment analysis of microarray data from Pimephales promelas (Rafinesque), a non-mammalian model organism. BMC Genomics. 2011;12(1):66.

36. Reich M, Liefeld T, Gould J, Lerner J, Tamayo P, Mesirov JP. GenePattern 2.0. Nat Genet. 2006;38(5):500-1.

37. Fontaneto $D$, Melone $G$. Do rotifer jaws grow after hatching? Hydrobiologia. 2005;546(1):213-21.

38. de Magalhães J, Curado J, Church GM. Meta-analysis of age-related gene expression profiles identifies common signatures of aging. Bioinformatics. 2009;27(7):875-81.

39. Glass D, Viñuela A, Davies MN, Ramasamy A, Parts L, Knowles D, Brown AA Hedman ÅK, Small KS, Buil A, et al. Gene expression changes with age in skin, adipose tissue, blood and brain. Genome Biol. 2013;14:R75.

40. Fushan AA, Turanov AA, Lee S-G, Kim EB, Lobanov A, Yim SH, Buffenstein R, Lee $S-R$, Chang K-T, Rhee $H$, et al. Gene expression defines natural changes in mammalian lifespan. Aging Cell. 2015;14(3):352-65.

41. López-Otín C, Galluzzi L, Freije José MP, Madeo F, Kroemer G. Metabolic control of longevity. Cell. 2016;166(4):802-21.

42. Stanfel MN, Shamieh LS, Kaeberlein M, Kennedy BK. The TOR pathway comes of age. Biochim Biophys Acta. 2009;1790(10):1067-74.

43. Biteau B, Karpac J, Hwangbo D, Jasper H. Regulation of Drosophila lifespan by JNK signaling. Exp Gerontol. 2011;46(5):349-54.

44. Katewa SD, Kapahi P. Role of TOR signaling in aging and related biological processes in Drosophila melanogaster. Exp Gerontol. 2011;46(5):382-90.

45. Guarente L, Kenyon C. Genetic pathways that regulate ageing in model organisms. Nature. 2000;408:255-62.

46. David DC, Ollikainen N, Trinidad JC, Cary MP, Burlingame AL, Kenyon C. Widespread protein aggregation as an inherent part of aging in C. Elegans. PLoS Biol. 2010;8(8):e1000450.

47. Copes N, Edwards C, Chaput D, Saifee M, Barjuca I, Nelson D, Paraggio A, Saad P, Lipps D, Stevens Jr SM, et al. Metabolome and proteome changes with aging in Caenorhabditis elegans. Exp Gerontol. 2015;72:67-84.
48. Ferguson AA, Roy S, Kormanik KN, Kim Y, Dumas KJ, Ritov VB, Matern D, Hu PJ, Fisher AL. TATN-1 mutations reveal a novel role for tyrosine as a metabolic signal that influences developmental decisions and longevity in Caenorhabditis elegans. PLoS Genet. 2013;9(12):e1004020.

49. La Du BN, Zannoni VG, Laster L, Seegmiller JE. The nature of the defect in tyrosine metabolism in alcaptonuria. J Biol Chem. 1958;230:251-60.

50. Feeney MB, Schöneich C. Tyrosine modifications in aging. Antioxid Redox Signal. 2012;17(11):1571-9.

51. Koike M, Shibata M, Ezaki J, Peters C, Saftig P, Kominami E, Uchiama Y. Differences in expression patterns of cathepsin C/dipeptidyl peptidase I in normal, pathological and aged mouse central nervous system. Eur J Neurosci. 2013;37(5):816-30.

52. Pawelec G, Goldeck D, Derhovanessian E. Inflammation, ageing and chronic disease. Curr Opin Immunol. 2014;29:23-8.

53. Howcroft TK, Campisi J, Louis GB, Smith MT, Wise B, Wyss-Coray T, Augustine $A D, M c E l h a n e y ~ J E$, Kohanski R, Sierra F. The role of inflammation in age-related disease. Aging. 2013;5(1):84-93.

54. Nikoletopoulou V, Tavernarakis N. Calcium homeostasis in aging neurons. Front Genet. 2012;3:200

55. Murchison D, Griffith WH. Age-related alterations in caffeine-sensitive calcium stores and mitochondrial buffering in rat basal forebrain. Cell Calcium. 1999:25(6):439-52.

56. Xiong J, Toescu EC VAF, Toescu EC. Changes in mitochondrial status associated with altered $\mathrm{Ca} 2+$ homeostasis in aged cerebellar granule neurons in brain slices. J Neurosci. 2002;22(24):10761-71.

57. Khachaturian ZS. The role of calcium regulation in brain aging: reexamination of a hypothesis. Aging (Milan, Italy). 1989;1(1):17-34.

58. Matthews EA, Linardakis JM, Disterhoft JF. The fast and slow afterhyperpolarizations are differentially modulated in hippocampal neurons by aging and learning. J Neurosci. 2009;29(15):4750-5.

59. Landfield PW, Pitler T. Prolonged Ca2 +-dependent afterhyperpolarizations in hippocampal neurons of aged rats. Science. 1984;226(4678):1089-92.

60. Bezprozvanny I. Calcium signaling and neurodegenerative diseases. Trends Mol Med. 2009;15(3):89-100.

61. Mattson MP. Calcium and neurodegeneration. Aging Cell. 2007;6(3):337-50.

62. Kim B-M, Mirbahai L, Mally A, Kevin Chipman J, Rhee J-S, Lee J-S. Correlation between the DNA methyltransferase (Dnmt) gene family and genome-wide 5 -methylcytosine $(5 \mathrm{mC})$ in rotifer, copepod, and fish. Genes Genomics. 2016;38(1):13-23.

63. Landry J, Sutton A, Tafrov ST, Heller RC, Stebbins J, Pillus L, Sternglanz R. The silencing protein SIR2 and its homologs are NAD-dependent protein deacetylases. Proc Natl Acad Sci. 2000;97(11):5807-11.

64. Wang R, Nguyen M, Qin FX-F, Tong Q. SIRT2 deacetylates FOXO3a in response to oxidative stress and caloric restriction. Aging Cell. 2007:6:505-11.

65. Vaquero A, Scher MB, Lee DH, Sutton A, Cheng HL, Alt FW, Serrano L, Sternglanz R, Reinberg D. SirT2 is a histone deacetylase with preference for histone H4 Lys 16 during mitosis. Genes Dev. 2006;20(10):1256-61.

66. North BJ, Marshall BL, Borra MT, Denu JM, Verdin E. The Human Sir2 Ortholog, SIRT2, Is an NAD-Dependent Tubulin Deacetylase. Mol Cell. 2003;11:437-44.

67. Barber MF, Michishita-Kioi E, Xi Y, Tasselli L, Kioi M, Moqtaderi Z, Tennen RI, Paredes S, Young NL, Chen K, et al. SIRT7 links H3K18 deacetylation to maintenance of oncogenic transformation. Nature. 2012;487(7405):114-8.

68. Ashraf N, Zino S, Macintyre A, Kingsmore D, Payne AP, George WD, Shiels PG. Altered sirtuin expression is associated with node-positive breast cancer. $\mathrm{Br} J$ Cancer. 2006;95(8):1056-61.

69. Hall JA, Dominy JE, Lee Y, Puigserver P. The sirtuin family's role in aging and age-associated pathologies. J Clin Invest. 2013;123(3):973-9.

70. Holdeman R, Nehrt S, Strome S. MES-2, a maternal protein essential for viability of the germline in Caenorhabditis elegans, is homologous to a Drosophila Polycomb group protein. Development. 1998;1245:2457-67.

71. Sen P, Shah Parisha P, Nativio R, Berger Shelley L. Epigenetic mechanisms of longevity and aging. Cell. 2016;166(4):822-39.

72. Benayoun BA, Pollina EA, Brunet A. Epigenetic regulation of ageing: linking environmental inputs to genomic stability. Nat Rev Mol Cell Biol. 2015; 16(10):593-610.

73. Hossain D, Tsang WY. Centrosome dysfunction and senescence: coincidence or causality?. J Aging Sci. 2013;1:113. doi:10.4172/2329-8847.1000113.

74. Oshima S, Seyama A. Cellular aging and centrosome aberrations. Ann N Y Acad Sci. 2010;1197:108-17.

75. Schatten $\mathrm{H}$, Chakrabarti A, Hedrick J. Centrosome and microtubule instability in aging Drosophila cells. J Cell Biochem. 1999;74(2):229-41. 